\title{
Comparison of 2-D Algorithms in EIT based Image Reconstruction
}

\author{
Vidya Sarode \\ Xavier Institute of Engineering, \\ Mumbai, India.
}

\author{
Sneha Patkar \\ Electrical Department, \\ VJTI, Mumbai, India.
}

\author{
Alice N Cheeran \\ Electrical Department, \\ VJTI, Mumbai, India.
}

\begin{abstract}
In Electrical Impedance Tomography (EIT), the resulting voltages developed by injecting a constant electrical current at the boundary electrodes are used to obtain images representing conductivity. Electrical Impedance and Diffused Optical Reconstruction Software (EIDORS) is used in this tomographic imaging. This paper compares different reconstruction algorithms used in EIT experiments conducted on a circular plastic phantom. Priors like Noser, Laplace, Tikhonov, Total Variation (TV) and Gauss-Newton and Back Projection algorithms are used for detection of nonconducting impurity. The images thus obtained are compared with respect to shape and size of impurity and perseverance of image contrasts.
\end{abstract}

\section{General Terms}

Electrical Impedance Tomography, Reconstruction Algorithms, Image Contrast

\section{Keywords}

Priors, Regularization, Impurities, Jacobian

\section{INTRODUCTION}

Tomographic imaging is a diagnostic modality that gives doctor a 2D cross-section view of a patient's body. 'Hardfield' techniques, such as X-ray, computed tomography (CT), magnetic resonance imaging (MRI) are very expensive. The machines are huge and bulky, so they are installed in a large, fixed place at a diagnostic center. Therefore they are unsuitable for bedside monitoring and imaging patients at home. Such scanners have side effects due to radiation. They cannot be used for emergency in ICU departments, or for infants [1]. 'Soft-field' modalities like EIT are intrinsically different compared to 'hard-field' techniques. As a noninvasive, nonradiating, nonionizing, and less expensive methodology, EIT is extensively used in clinical diagnosis, biotechnology, and other nondestructive applications [2, 3]. EIT has wide applications in imaging breast and brain tumors, cancer detection and lung functioning. Though EIT image has low spatial resolution, high temporal resolution makes this technique a research area.

EIDORS is used for 2D and 3D reconstruction of image based on voltage dataset. Its goal is to provide freely distributable and modifiable software for image reconstruction [4]. It is implemented in a MATLAB package for two-dimensional mesh generation, solving forward problem, reconstruction using inverse models and display of the images. To perform the EIT experiment directly on the patient's body is neither ethical nor permitted hence initial work has been carried out with phantoms.

The reconstruction of images using different algorithms is explained in subsequent sections. Section 2 describes the general EIT system and experimental setup. The algorithms used for reconstruction are explained in section 3. Section 4 shows the reconstructed images, and compares them with different algorithms. Section 5 summarizes and throws light on future scope.

\section{ELECTRICAL IMPEDANCE TOMOGRAPHY (EIT)}

EIT reconstructed image is a mapping of conductivity distribution, by means of measurement of voltages [5] at the surface of object made by the injection current to media (Figure 1). The surface potential depends on the current flux, which depends on the material profile. By studying the boundary potential data, an internal material profile can be reconstructed.

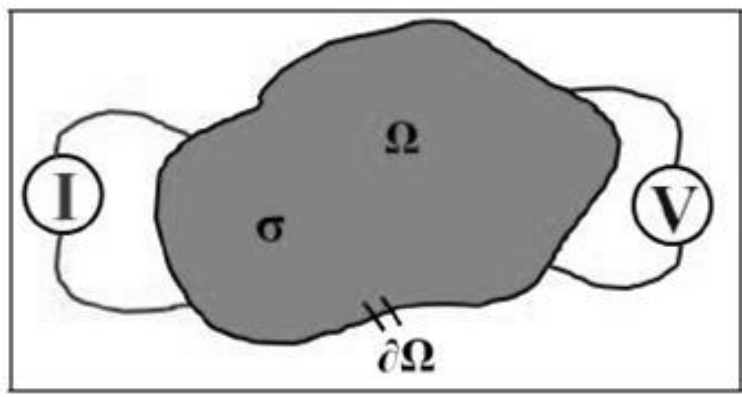

Figure 1: General EIT system

In EIT, the field strength at any point is a non-linear function of the distribution of the electrical properties throughout the body. The spatial resolution of the reconstructed images is severely affected by the ill-posedness of the image reconstruction problem [6]. With some prior knowledge of the distribution, inverse solution can be implemented. Regularization techniques used vary in their complexity depending on the assumptions they seek to enforce. They include smoothing priors as well as priors preserving sharp discontinuities.

Experimental setup consists of signal generator, a voltage to current converter $(20 \mathrm{~mA}$ at $5 \mathrm{KHz})$, a closed phantom $(14 \mathrm{~cm}$ diameter and $7 \mathrm{~cm}$ height) with 16 gold plated silver electrodes. Experiments performed on plastic phantom filled with saline solution and 16 electrodes mounted on the boundary equidistantly [7]. The constant sinusoidal current is applied to the pair of electrodes in neighboring pattern as shown in figure 2 . In this method, the current is applied through neighboring electrodes and the voltage is measured successively from all other adjacent electrode pairs. Some other current injection patterns are opposite, cross and adaptive $[8,9]$. 


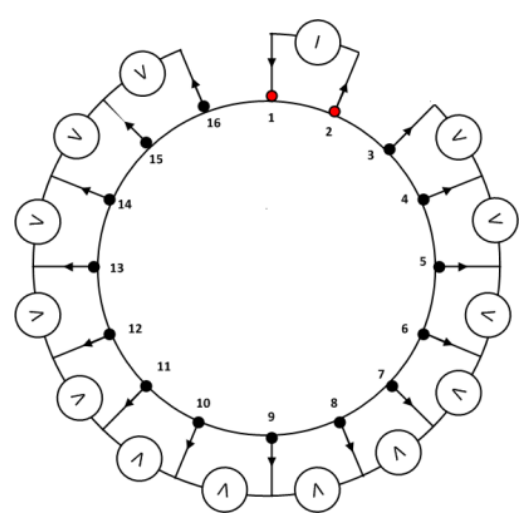

Figure 2: Adjacent Current Injection Method

The 16 electrodes are arranged inside phantom in clockwise direction. An adjacent stimulation pattern is used with no measurement taken at current stimulation electrodes, giving $\boldsymbol{M}=\boldsymbol{E} \times(\boldsymbol{E}-\mathbf{3})$, where $\boldsymbol{M}=$ total number of measurements per frame and $\boldsymbol{E}=$ number of electrodes. For a 16 electrode system, this gives 208 measurements. Two voltage datasets obtained without impurity and with impurity are used for reconstruction of resistivity images.

\section{RECONSTRUCTION ALGORITHMS}

Both static and dynamic imaging exists in EIT. The static imaging involves producing an image with absolute conductivity distribution, while the dynamic imaging reconstructs images of change in conductivity [10]

\subsection{Back-Projection Algorithm}

It is a linear reconstruction algorithm proposed by Barber and Brown [11] which is capable of producing images of changes in conductivity. However, the images produced by back projection have some clear artifacts because of inherent character of the asteroid trace. The conductivity equation given by Brown and Barber is:

$$
\delta \sigma=\frac{1}{m} \sum_{i=1}^{m} W\left(p_{i}\right)\left(2 V\left(p_{i}\right)-1\right)
$$

Where $\boldsymbol{m} \rightarrow$ number of electrodes

$$
\begin{aligned}
& \boldsymbol{W} \rightarrow \text { voltage measurement } \\
& \boldsymbol{P} \rightarrow \text { position of electrode } \\
& \boldsymbol{V} \rightarrow \text { current intensity function }
\end{aligned}
$$

The $\phi$ stands for boundary measurement vector and $\Delta \boldsymbol{\sigma}$ is the conductivity distribution inside the body. The Jacobean matrix is given by $\frac{\partial V_{s}}{\partial \sigma}=\boldsymbol{J}$. The relative difference in boundary measurement is given as

$$
\phi=\frac{\left[V_{s}-V_{m}\right]}{V_{s}}
$$

From definition of Jacobean Matrix

$$
\partial \phi=J X \partial \sigma
$$

Then change in conductivity is given as

$$
\begin{gathered}
\partial \sigma=J^{T} X \partial \phi \\
\partial \sigma=J^{T}\left[J^{T} J\right]^{-1} \partial \phi
\end{gathered}
$$

In Back Projection method $\boldsymbol{J}^{T}$ is replaced by Back Projection operator.

$$
\partial \sigma=B[B J]^{-1} \partial \phi
$$

$[\boldsymbol{B J}]^{-\mathbf{1}}$ shows filtration in boundary data and $\boldsymbol{B}[\boldsymbol{B J}]^{-\mathbf{1}}$ backprojects this data into an image. In filtered backprojection, the data with more accurate values is filtered through above algorithm which is then used for reconstruction.

\subsection{Gauss Newton Approach}

Electrical impedance imaging is a highly nonlinear and illposed inverse problem in which minimization algorithm is used to obtain its approximate solution [12]. Objective function is minimized by taking the difference between the experimental measured data and predicted data. If $\boldsymbol{V} \boldsymbol{m}$ is the measured voltage matrix and $\boldsymbol{V} \boldsymbol{c}$ is the calculated voltage matrix, then the Gauss-Newton (GN) algorithm gives a least square solution of the minimized object function $\boldsymbol{s}(\boldsymbol{\sigma})$ defined as:

$$
s(\sigma)=\frac{1}{2}\left\|V_{m}-V_{c}\right\|^{2}=\frac{1}{2}\left(V_{m}-V_{c}\right)^{T}\left(V_{m}-V_{c}\right)
$$

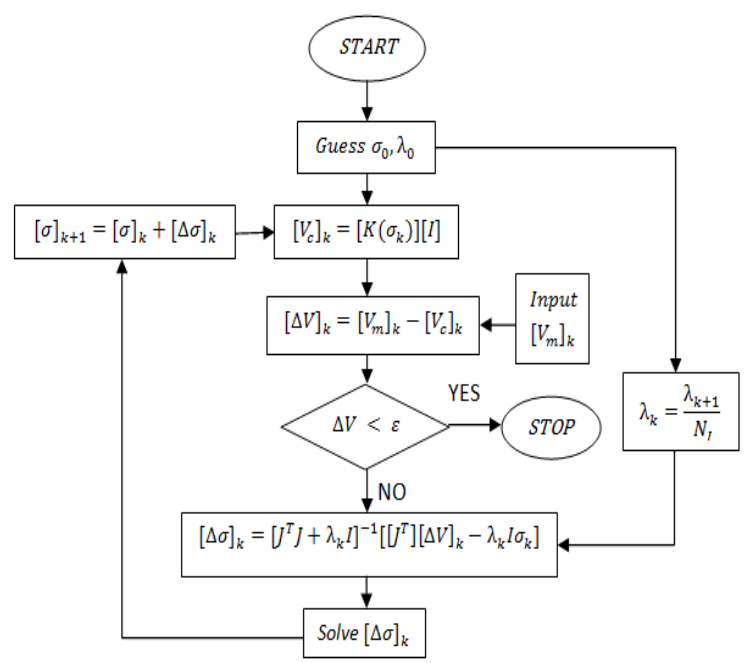

Figure 3: Flow chart of Gauss-Newton Algorithm

The algorithm starts with an initial conductivity $\boldsymbol{\sigma}_{\mathbf{0}}$ and the voltages are calculated which are compared with the measured potential data to obtain Jacobian $(\boldsymbol{J})$ matix. $\Delta \boldsymbol{\sigma}$ is then updated and $\boldsymbol{\sigma}_{\boldsymbol{k}+\boldsymbol{1}}$ is calculated using $\boldsymbol{\sigma}_{\boldsymbol{k}}$. This is repeated for number of iterations till specific error limit $\boldsymbol{\varepsilon}$ is reached, as shown in figure $3 . \lambda$ is the regularization parameter which is also updated in each iteration.

\subsection{Regularization Filters}

The inverse problem is formulated so as to minimize regularization functional [13]. The reconstruction problem is ill conditioned and regularization techniques are necessary in order to stabilize the process. Reconstructed conductivity is the solution of the least-square problem.

$$
s_{\text {rec }}=\operatorname{argmin}\|h(s)-z\|^{2}
$$

$$
\begin{aligned}
& \boldsymbol{z} \rightarrow \text { vector of measured voltages } \\
& \boldsymbol{s} \rightarrow \text { discrete conductivity } \\
& \boldsymbol{h} \rightarrow \text { nonlinear forward operator from model space to } \\
& \boldsymbol{s}_{\boldsymbol{r e c}} \rightarrow \text { measurements space }
\end{aligned}
$$

\subsubsection{Tikhonov Regularization}

It is a differential operator used as regularization functional, leading to smooth solutions.

$$
s_{r e c}=\operatorname{argmin}\left[\|h(s)-z\|^{2}+r^{2} F(s)\right]
$$

$\boldsymbol{r} \rightarrow$ regularization parameter 
$\boldsymbol{L} \rightarrow$ regularization matrix

$$
\begin{gathered}
F(s)=\|L s\|^{2} \\
s_{\text {rec }}=\operatorname{argmin}\left[\|h(s)-z\|^{2}+r^{2}\|L s\|^{2}\right]
\end{gathered}
$$

This regularization method achieves the stability of the inversion by penalizing sudden variations in the conductivity.

\subsection{Noser Algorithm}

This algorithm is based on the methods of least squares. It takes one step of a Newton's method, using a constant conductivity as an initial guess. Most of the calculations can therefore be done analytically. It is named NOSER for Newton's One Step Error Reconstructor [14]. This algorithm does not reproduce the conductivity accurately but useful images can be obtained.

Jacobian matrix is ill-conditioned, meaning some of its eigen values are large and some are very small. Because of small eigen values, if it is inverted, it will have very large eigen values. These large values would cause large errors. To overcome this problem, Matrix $\boldsymbol{J}$ is modified. The modified version of $\boldsymbol{J}$ is given by

$$
\begin{aligned}
\boldsymbol{J} & =\boldsymbol{A}_{n, m}+\boldsymbol{r} \boldsymbol{A}_{n, m} \delta_{n, m} \\
\text { where } \delta & =1, \text { when } \mathrm{n}=\mathrm{m} \\
& =\mathbf{0}, \text { otherwise }
\end{aligned}
$$

Matrix $\boldsymbol{A}$ has its largest elements on and near the diagonal. By taking large value of parameter ' $\boldsymbol{r}$ ', matrix $\boldsymbol{J}$ is forced to be diagonally dominant and thus conditioning of the matrix $\boldsymbol{J}$ is improved. The parameter ' $\boldsymbol{r}$ ' is selected using empirical methods to keep balance between stability and image contrast.

\subsection{Total Variation Reconstruction}

Reconstruction algorithm with regularization based on the Total Variation (TV) functional helps to preserve discontinuities in reconstructed profiles [15], which are smoothened by traditional reconstruction algorithms like Newton's algorithm. Quadratic regularization or backprojection algorithms are known to blur image contrasts. In cancer detection, it is important to detect small localized conductivity contrasts. Edge preserving algorithms like those using TV functional are more complex to implement. The total variation $(\boldsymbol{T V})$ of a conductivity image is defined as:

$$
T V(\theta)=\int|\nabla \theta| d \Omega
$$

Where $\boldsymbol{\Omega}$ is the region to be imaged. Since the conductivity is constant over each FEM element, $\boldsymbol{\nabla} \boldsymbol{\theta}$ is non-zero only on the edges between elements. For the $\boldsymbol{i}$-th edge, shared by the FEM elements $\boldsymbol{m}(\boldsymbol{i})$ and $\boldsymbol{n}(\boldsymbol{i})$, the jump in conductivity is: $\left|\boldsymbol{\theta}_{\boldsymbol{m}(\boldsymbol{i})}-\boldsymbol{\theta}_{\boldsymbol{n}(\boldsymbol{i})}\right|$. The variation over the complete image can be found by integrating this jump over all the edges of the mesh:

$$
\boldsymbol{T V}(\theta)=\sum_{i} l_{i}\left|\theta_{m(i)}-\theta_{n(i)}\right|
$$

Where $\boldsymbol{l}_{\boldsymbol{i}}$ is the length of $\boldsymbol{i}$-th edge in the mesh, and the index $\boldsymbol{i}$ covers all the edges. Above equation can be expressed in terms of matrices as:

$$
T V(\theta)=\sum_{i}\left|L_{i} \theta\right|
$$

Where $\boldsymbol{L}$ is a sparse matrix with one row per each edge. Every row $\boldsymbol{L}_{\boldsymbol{i}}$ has two non zero elements in the columns $\boldsymbol{m}(\boldsymbol{i})$ and $\boldsymbol{n}(\boldsymbol{i})$ :

$$
L_{i}=\left[0, \ldots, 0, l_{i}, 0, \ldots, 0,-l_{i}, 0 \ldots 0\right]
$$

TV regularization is therefore of the $\boldsymbol{L 1}$ kind since it is a sum of absolute values. Such regularization does not penalize image discontinuities.

\section{COMPARISON OF ALGORITHMS}

The non conducting impurity used for experiments is clay cylinder of $18 \mathrm{~mm}$ diameter and $57 \mathrm{~mm}$ height. It is placed near electrodes 4 and 5 inside the plastic phantom containing saline solution. First aim is to create finite element model of electrodes and circular container using EIDORS. Then the voltage data set obtained with adjacent current pattern is fed in the MATLAB excel sheet. Images are reconstructed using different algorithms. Non conducting impurity is identified by red color in EIDORS symbolizing high voltage. Whereas blue color symbolizes low voltage, identifying conducting impurity.

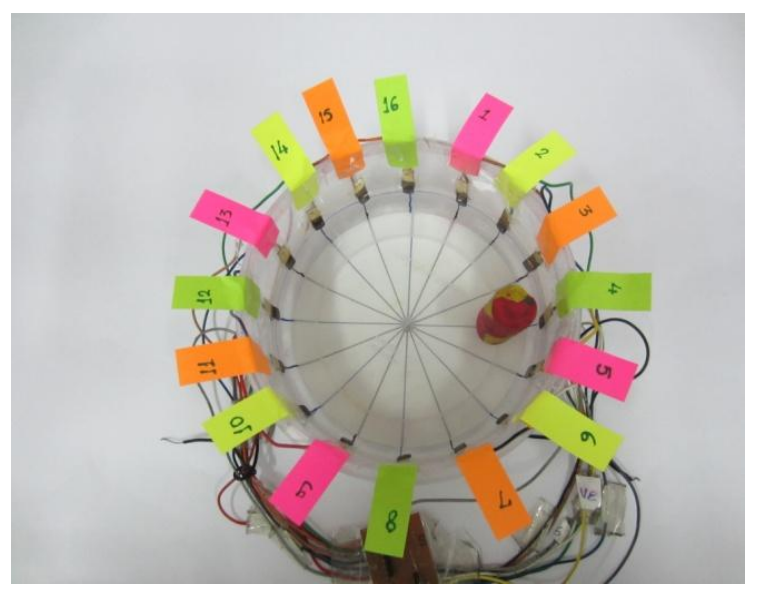

Figure 4: Clay cylinder kept near electrodes 4 and 5

\subsection{Back Projection Algorithm}

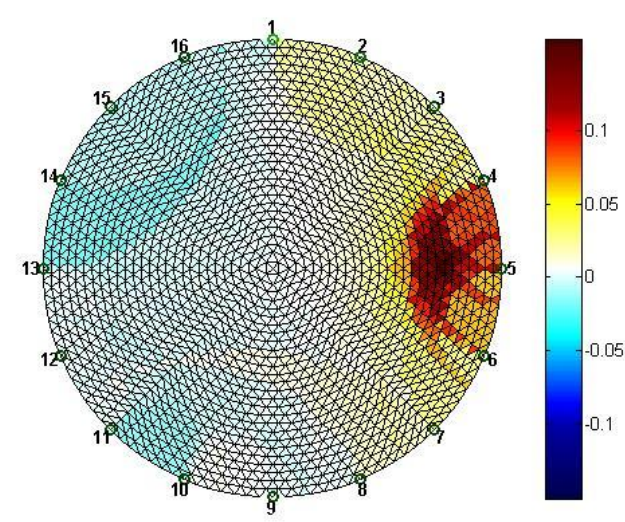

Figure 5: Non conducting impurity near 4, 5 using Back Projection without filter 


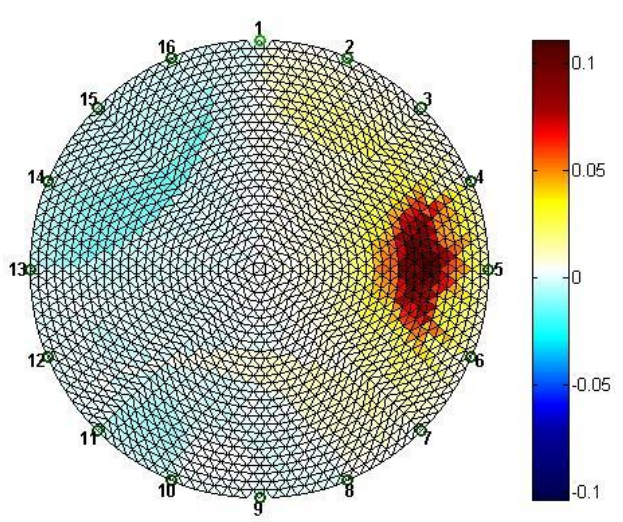

Figure 6: Non conducting impurity near 4, 5 using Back Projection with filter

Back projection algorithm is able to find the location and nature of impurity (Figure 5). But clear artifacts are seen in image because of inherent character of asteroid trace shown by direct back project. In filtered back projection, data with more accurate values are filtered and then this data is used for reconstruction to get clearer images, as seen in figure 6 . Disadvantage is that, back projection algorithm blurs the image contrasts.

\subsection{Gauss-Newton Algorithm}

Different priors can be used to find images of conductivity with the help of GN algorithm.

\subsubsection{Using Tikhonov Prior}

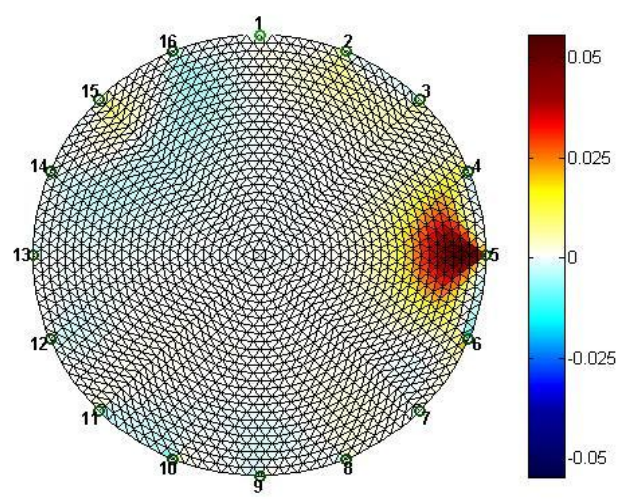

Figure 7: GN algorithm with Tikhonov prior

In Tikhonov regularization, differential operators are used, which leads to smooth solutions. It stabilizes the reconstruction process but imposes a penalty on non-smooth regions in a reconstructed image. Discontinuities in reconstructed profiles are smoothened as seen in figure 7 . The circular shape of the cylinder is more clearly seen as compared to back-projection algorithm but not as accurately as Laplace and TV priors.

\subsubsection{Using Noser Prior}

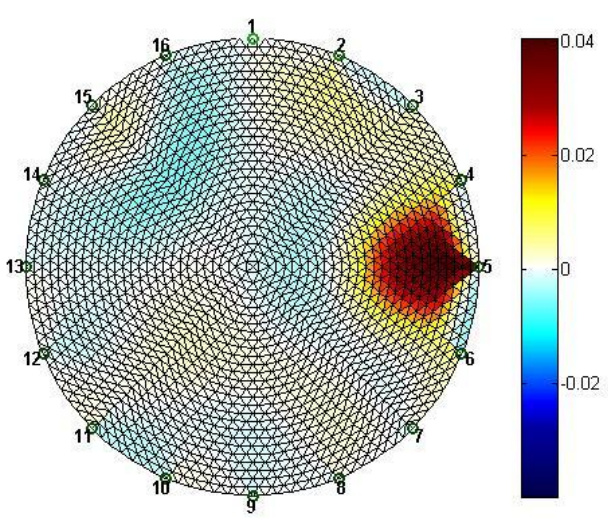

Figure 8: GN algorithm with Noser prior

This prior is $\operatorname{diag}\left(\operatorname{diag}\left(\mathrm{J}^{\prime} * \mathrm{~J}\right)^{\wedge}\right.$ exponent $)$, where $\mathrm{J}=\mathrm{Jacobian}$ matrix. This algorithm does not reproduce the conductivity accurately. This is because Jacobian matrix is ill-conditioned and value of exponent needs to be chosen correctly to keep balance between stability and image contrast. The image reconstructed is not as sharp as obtained with Laplace or TV prior. Figure 8 shows that the high voltage region is spreaded to larger area (electrodes $4,5,6$ ).

\subsubsection{Using Laplace Prior}

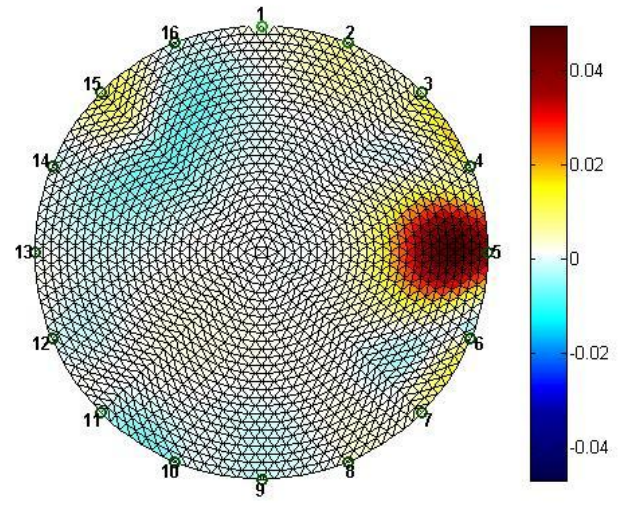

Figure 9: GN algorithm with Laplace prior

The Laplace prior is a 2 nd order high pass filter. On a finite element mesh, it is defined as -1 for each adjacent element, and 3 (in 2D) or 4 (in 3D) for the element itself. Hence sharper reconstruction is possible as compared to back projection or NOSER algorithm. Laplace is an edge preserving algorithm. Figure 9 shows reconstructed image with sharp discontinuities in conductivity. The shape is more accurately reconstructed as compared to Tikhonov and Noser priors. 


\subsection{Total Variation (TV) Reconstruction}

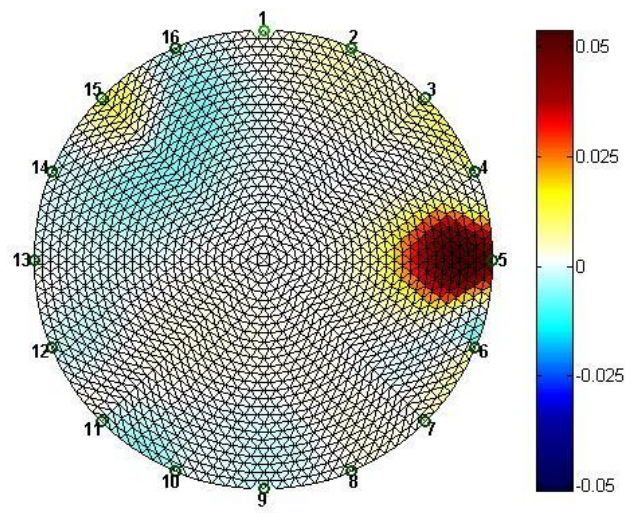

Figure 10: Total Variation Reconstruction

From figure 10, it can clearly be seen that discontinuities are preserved. The ability of reconstructing sharp changes leads to a better estimation of the boundaries and also to a better accuracy in the estimated values. Thus TV regularization shows improved ability to reconstruct sharp contrasts. High voltage region symbolizing low electric conductivity is more confined as compared to other priors used. And circular shape of clay cylinder is more clearly seen by brown color. But this algorithm is more complex to implement.

Table 1: Comparison of actual and reconstructed impurity size

\begin{tabular}{|c|c|c|}
\hline $\begin{array}{c}\text { Impurity (Clay } \\
\text { cylinder) }\end{array}$ & $\begin{array}{c}\text { Size } \\
\left(\mathbf{c m}^{\mathbf{2}}\right)\end{array}$ & $\begin{array}{c}\text { Error (\%) } \\
=\end{array}$ \\
$\frac{\mathbf{4 . 5 5 9 9}^{*} \text {-reconstructed } \mid}{\mathbf{4 . 5 5 9 9}}$ \\
\hline $\begin{array}{c}\text { Reconstructed using } \\
\text { Tikhonov prior }\end{array}$ & 3.619 & $20.63 \%$ \\
\hline $\begin{array}{c}\text { Reconstructed using } \\
\text { Noser prior }\end{array}$ & 5.2285 & $14.66 \%$ \\
\hline $\begin{array}{c}\text { Reconstructed using } \\
\text { Laplace prior }\end{array}$ & 4.84946 & $6.35 \%$ \\
\hline $\begin{array}{c}\text { Reconstructed using } \\
\text { TV prior }\end{array}$ & 4.7047 & $3.175 \%$ \\
\hline
\end{tabular}

* Actual size of clay cylinder is $4.5599 \mathrm{~cm}^{2}$

With 3136 mesh elements (used in forward model specifications), Tikhonov prior gives smaller size than actual size of the impurity whereas reconstructed size of clay cylinder by Noser prior is bigger than actual. Laplace and TV priors obtain reconstructed size very close to the actual one. The percentage error is minimum for TV reconstruction.

\section{CONCLUSION AND FUTURE SCOPE}

EIT based reconstruction is a very convenient alternative to traditional, radiating, expensive imaging techniques to yield useful images. It is nonradiating, noninvasive, cost effective approach making it useful for imaging breast and brain tumors etc. The conductivity distribution depends on the material of electrodes, number of electrodes and the medium used in experiment. Different algorithms used for reconstruction are compared for nonconducting impurity in a closed phantom.
Location of impurity can be guessed easily using back projection. But it gives clear artifacts, thus shape of impurity is difficult to observe. To overcome the asteroid trace, mean back-projection algorithm can be used. Gauss-Newton approach yields better images with respect to shape and size of impurity. As compared to other priors, Total Variation (TV) prior gives better circular shape and size of clay cylinder and also sharper image is obtained using EIDORS. Conducting impurity can be reconstructed in a similar way. Presence of more than 1 impurity can also be detected. The number of mesh elements in the forward model used affects the size and shape of impurity, which can be studied in future.

\section{ACKNOWLEDGEMENTS}

The authors thank Xavier Institute of Engineering and VJTI. This work was not possible without the help of Fr Reginald Tauro, Director XIE and Fr Fabian Barreto, Administrator XIE.

\section{REFERENCES}

[1] Tushar K. Bera, J Nagaraju, "Electrical Impedance Tomography (EIT) System for Radiation-Free Medical Imaging Based on LabVIEW", pp.: 1-4.

[2] Bera T. K and Nagaraju J., "Resistivity Imaging of a reconfigurable phantom with circular inhomogeneities in 2D-electrical impedance tomography", Measurement 44(2011), pp 518-526, doi:10.1016/j.measurement .2010 .11 .015

[3] Erwati M. T, and Farrukh N., "Applications of electrical impedance tomography for imaging in biomedical and material technology", Proceedings of 2009 IEEE Students Conference on research and development, Nov '09.

[4] Andy Adler and William R.B. Lionheart, "Uses and abuses of EIDORS: An extensible software base for EIT", The University of Manchester, May 2006.

[5] Amir Movafeghi, Ali R. Nateghi, ManouchehrSoleimani, Mohammad H. Kargarnovin and Hamid Soltanianzadeh, "Image Reconstruction Algorithms or Sut-1 Eit System", pp.: 579-582, 2004.

[6] Nicholas Polydorides, "Image Reconstruction Algorithms for Soft-field tomography", Manchester, United Kingdom, September 2002.

[7] VidyaSarode, Priya M. Chimurkar, Alice N Cheeran, "Electrical Impedance Tomography using EIDORS in a Closed Phantom", International Journal of Computer Applications (0975 - 888), Volume 48- No.19, June 2012.

[8] TusharKantiBera and J. Nagaraju, "A Study of Practical Biological Phantoms with Simple Instrumentation for Electrical Impedance Tomography (EIT)", I2MTC, Singapore, 5-7 May 2009.

[9] Bera T. K and Nagaraju J., "Studying the resistivity imaging of chicken tissue phantoms with different current patterns in Electrical Impedance Tomography (EIT)", Measurement (2012), doi:10.1016/j.measurement .2012 .01 .002 . 
[10] Zou Y. and Guo Z., "A review of electrical impedance techniques for breast cancer detection", Elsevier Medical Engineering and Physics 25, (2003).

[11] D.C. Barber, N. J. Avis, "Single Step Algorithms for Image Reconstruction", Electrical Impedance Tomography Applied Potential Tomography IEE Colloquium on (1992), pp 4/1-4/3.

[12] TusharKantiBera, J. Nagaraju, "A Chicken Tissue Phantom for Studying an Electrical Impedance Tomography (EIT) System Suitable for Clinical Imaging", Sens Imaging (2011) 12:95-116 DOI 10.1007/s11220-011-0063-4.
[13] Andrea Borsic, William R. B. Lionheart, and Christopher N. McLeod, "Generation of AnisotropicSmoothness Regularization Filters for EIT", IEEE Transactions on Medical Imaging, Vol. 21, No. 6, June 2002.

[14] M. Cheney and D. Isaacson, "NOSER: An Algorithm for Solving The Inverse Conductivity Problem", International Journal of Imaging Systems and Technology, Vol.2, 66-75 (1990).

[15] Borsic, B. M. Graham, A. Adler, W. R. B. Lionheart, "In vivo Impedance Imaging with Total Variation Regularization”, pp.: 1-10. 